\title{
$X$. On the theory of congeneric surd equations
}

\section{W.G. Horner Esq.}

To cite this article: W.G. Horner Esq. (1836) X. On the theory of congeneric surd equations, Philosophical Magazine Series 3, 8:43-44, 43-50, DOI: 10.1080/14786443608648795

To link to this article: http://dx.doi.org/10.1080/14786443608648795

曲 Published online: 21 Aug 2009.

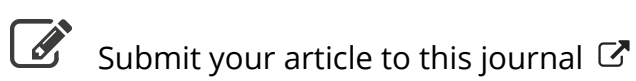

Џ Article views: 2

Q View related articles $₫$ 
X. On the Theory of Congeneric Surd Equations. By W. G. Horner, Esq. (In a Letter addressed to T. S. Davies, Esq., F.R.S. L.\&.E.)*

[If those mathematicians who have met with a quadratic equation $\uparrow$ whose "roots" either under a real or imaginary form could not be exhibited, will recall to inemory the surprise with which they viewed the circumstance, and the attempts which they made to solve the mystery, they will read with no ordinary gratification the following discussion of the general question of which this forms a part. The general theory of such equations, very happily named by Mr. Horner " Congeneric Equations," is here laid down with great clearness, and, so far as I know, for the first time, - as it is, indeed, nearly the first time the formation of any general and philosophic views respecting them has been attempted.

The following letter was drawn up in answer to some passages in one which $\mathrm{I}$ had a short time previuusly addressed to $\mathrm{Mr}$. Horner, and was a private and friendly communication; yet I have sincere pleasure in having obtained his permission to publish it in the Phil. Mag. I do so under the conviction that it will furnish the same satisfaction to others that it has done to me. I shall only add in conclusion my hope that the inquiry which, in the close of his letter, he has assigned to me, will be pursued by himself, as I know no man to whom such researches can be so safely and successfully referred.

Royal Military Academy, Nov. 15, 1835.

T. S. D.]

\section{My dear $S_{1 R}$,}

AGREE with you in thinking that the properties of irrational equations have not received that degree or kind of attention from writers on the elements of algebra, which was due either to the importance of the subject, or to a consideration for the comfort of young students. This appears the more extraordinary, because the methods of clearing an equation from irrational expressions, whether involving the unknown or not, have been so fully discussed, that really very little remained to be done for rendering the state of the whole case very intelligible. Waring (Med. Alg., Prob. 26.) may be cited as a case in point. But "a miss is as good as a mile." In solving equations involving radicals every one has experienced the necessity of putting his results to the proof before he could venture to decide which of them, or whether any of them, could be trusted; but as the latter alternative, or the failure of every result, is of rare occurrence in books of

* Communicated by Mr. Davies.

+ For instance, $2 x+\sqrt{x^{2}-7=5}$, the "roots" of which are 4 and $\frac{8}{3}$ as determined by the common process; neither of which substituted in the equation reduces it to zero. These are the roots of its congeneric surd cquation $2 x-\sqrt{x^{2}-7}=5$. 
exercises, because, no doubt, the compilers had not thought the matter out, we who use their collections, being as indolent as they, have contented ourselves with the general probability of, at least, partial success. In the mean time even classical writers have spoken of clearing an equation from radicals, in order to its solution, as a process of course, and which would not in any way affect the conditions. The consequence is, that a habit prevails of talking about equations without any regard to this peculiar case, and therefore in language which when applied to it becomes quite incorrect. The term root of an equation passes for synonymous with any quantity which, being substituted for the unknown, satisfies the conditions; and it is affirmed, and demonstrated, that every equation has at least one root; and that, having one, it must have as many roots as there are units in the greatest index attached to the unknown. It is therefore quite startling, when we are reminded that equations may be proposed ad libitum, whose conditions cannot be satisfied by any quantity, positive, negative, or imaginary; that notwithstanding this, the roots obtained from such equations may be real quantities. Nor is the enigma solved by discovering that the roots obtained from one equation are sure to satisfy the conditions of another, not much unlike it: on the contrary, one is quite displeased at this kind of thimble-rig shuffling, where we were assured of finding truth, the whole truth, and nothing but the truth. A logician of the old school would settle the business by crying "distinguo"; but we should still reply, that it is a lame distinction which clears up only one half of the premises: we know that these are surd equations we are now speaking of, and that just before we were speaking of rational equations, or equations cleared of surds; but the difficulty remains unexplained. If he really knew a little of the subject, he would, perhaps, next try the pass-word ambiguity: "There is always a certain ambiguity adhering to surd expressions." When, however, the most is said that can be said to that purport, it amounts in short to this, that in the reading of formulæ, when we meet with a radical, we ought not to use the definite but the indefinite article. We have a knack of saying "the", where we ought to say " $a$ ", that is all; and if we did but read $a$ square root, $a$ cube root, and so on, we should be certain of finding one that would satisfy the existing conditions. This sounds plausibly, and at least ninety. nine out of every hundred of algebraists would inquire no further; but you would perhaps object, that at this rate $+\sqrt{ } x=-\sqrt{x}$ might be a good equation, unless, with 
Lindley Murray, we admit the "the" when the same quantity appears a second time under the same radical; and that without a greater latitude still, we should never be able to prove that $+\sqrt{x-a} \times-\sqrt{x+a}$ made $-\sqrt{x^{2}-a^{2}}$, and so on. So that the professed ambiguity is subject, after all, to a conventional permanence.

The source of the whole mystery, in my judgement, is to be found in the almost unavoidable imperfection of the manner in which we are taught to transform equations when we are at school. The operations consequent on transposition are correct as far as their principles are resolvable into Euclidian axions. Beyond that they are liable to fallacy; and, generally speaking, we are infallible in our judgement only as long as every term is on one side. We may then determine satisfactorily in what cases zero is admissible as the aggregate value. An instance of the hazard attending the neglect of this principle is given in my paper in the Lond. and Edinb. Phil. Mag. for September 1894, (vol.v.) p. 189. In the management of surds, instances might easily be accumulated.

And whence this hazard? and why consequent upon transposition? Because, from the nature of analysis, we are continually arguing from the direct to the converse. An equation is formed hypothetically. We trace out certain direct consequences, in the form of equations also, and so on; until an equation is obtained, such that if the first be true, the last is therefore true. But the converse is that which we wished to ascertain. Is the hypothetical equation true, because the resulting equation is so? To determine this, a similar query must be instituted from link to link throughout the chain of reasoning. Is each equation in succession true, because the next in succession is so? If each of these subordinate inquiries admits of a decided affirmative, the reply to the general query is satisfactory; otherwise, it is not. Now in the management of equations, we have been taught, either virtually or in direct terms, to rely upon certain axioms, which for the present purpose will be most effectually stated in pairs, viz.

If equals be added to equals, the wholes are equal;

If equals be subtracted from equals, the remainders $\}$ I.

If equals be multiplied by equals, the products are? equal; and equal; and
If equals be divided by equals, the quotients are II. 
If equals be raised to powers denoted by equal ex-? ponents, the powers are equal; and

If of equal quantities rots be extracted, which $>$ III. are denoted by equal exponents, the roots are equal.

It has never been my chance, either to hear the validity of any of these principles called in question, or even any caution suggested as necessary in the application of them; and yet, when tested by the combined trial of their direct and reflex action, they will presently appear to be very susceptible of misuse in incautious hands.

The first and second pair, abstractedly considered, afford such entire conviction, that in each of them, if either proposition is granted, the other can be strictly demonstrated by means of it; and the second pair are truly corollaries to the first. No hesitation, no ambiguity, is felt.

The fifth proposition, as a clear corollary to the third, is, in itself, equally satisfactory; but quite otherwise in regard to its reflex effect, as described in the sixth. For, being aware that if unequal quantities $(+a,-a)$ be raised to power, denoted by equal exponents, the powers may nevertheless be equal; we are assured that, conversely, if of equal quantities roots be extracted which are denoted by equal exponents, the roots may nevertheless be unequal.

This remark furnishes a sufficient reason for rejecting the third pair of principles, and consequently the ordinary method of clearing an equation from surds. For, in every instance in which this is effected by transposition and involution, in compliance with the fifth axiom, we tacitly assume that such step can be retraced with equal certainty by means of the sixth; whereas, in any such transit, the consequent equation may be quite true, and yet the antecedent be quite false.

If, however, we attribute the failure of the third pair of axioms to a special ambiguity peculiar to evolution, we shall remain under a delusion, and miss the cause and remedy of the evil. Involution is but a single instance of the erroneous application of the axioms of the third pair ; but the use of any of the four unexceptionable axioms is liable to be frustrated by a similar cause, although in some cases the absurdity introduced is so palpable as to occasion a kind of instinctive unconscious avoidance. In other instances, however, even acute minds have failed to observe the fallacy. This I shall now point out, and prove that unless connected with the use of the first pair of axioms, it will be avoided, if no member of the equation is transposed to the zero side.

The origin of the fallacy in question will be rendered more 
evident by a course of amusing experiments upon a familiar equation, $e . g$.

$$
x^{4}+2 x^{3}-7 x^{2}-8 x+12=0,
$$

whose roots are $1,8,-3,-2$. Applying the four axioms in succession, we shall perceive how the incautious blending of two truths, by means of rules in themselves unexceptionable, will produce a falsehood.

$$
\begin{aligned}
& \text { 1st. To } x^{4}+2 x^{3}-7 x^{2}-8 x+12=0 \\
& \text { Add } \begin{array}{r}
x-1=0 \\
x^{4}+2 x^{3}-7 x^{2}-7 x+11=0 ;
\end{array}
\end{aligned}
$$

a false equation, with regard to all the values of $x$, with the single exception of 1 , the value already used. Similar results would accrue from the addition or subtraction of any other divisor of the equation; the result will be false in every value, except those which are also found in the equation added or subtracted. Thus,

$$
\begin{aligned}
& \text { From } x^{4}+2 x^{3}-7 x^{2}-8 x+12=0 \\
& \text { Take } \frac{6 x^{2}-18 x+12=0}{x^{4}+2 x^{3}-13 x^{2}+10 x=0}
\end{aligned}
$$

whose only correct roots are those also of $x^{2}-3 x+2=0$.

2ndly, The given equation is resolvable into the quadratics $x^{2}-3 x+2=0$, and $x^{2}+5 x+6=0$.

Therefore, multiply $\quad x^{2}-3 x=-2$

$$
\text { by } \frac{x^{2}+5 x=-6}{x^{4}+2 x^{3}-15 x^{2}=-12 ;}
$$

a statement altogether erroneous, not containing a single correct value of $x$.

On the other hand, divide

$$
\begin{aligned}
& x^{4}+2 x^{3}-7 x^{2}-8 x=-12 \\
& \text { by } x^{2}-3 x=-2 ; \\
& \therefore \frac{x^{3}+2 x^{2}-7 x-8}{x-3}=6, \\
& \therefore x^{3}+2 x^{2}-13 x=-10:
\end{aligned}
$$

incorrect, except in respect of the roots of $x^{2}-3 x+2=0$.

You will clearly perceive, without dwelling upon the distinction of cases, the very simple nature and origin of the paradox. The axioms speak of quantities which are simultaneously equal; but no two roots of an equation, unless they be equal roots, are coexistent : if $x=1$ it is not at the same time $=2$. Consequently, as in each of the examples $x$ in the upper of the two equations has some values, which substituted in the 
lower will render its sides unequal, the results, as far as such values of $x$ are concerned, are no longer coincident with the conditions of the axioms on which the management of equations is founded, but are illustrations of the opposite axioms, viz. that unequals added to, or subtracted from, or multiplied or divided by, equals, produce unequal results, or in algebraic language, false equations.

The reason why this inconvenience, in the use of the second pair of axioms, cannot occur when all the terms are on one side and zero alone on the other, is very evident; although, by another of those paradoxes by which equations are beset, the complete truth appears at first sight to be the result of combining a truth with an error, and equals to result from combining equals with unequals. It is, however, easy to avoid all suspicion of error. Thus, it was said, that the given equation is resolvable into $x^{2}+3 x+2=0$, and $x^{2}+5 x+6=0$. But as these statements are not simultaneously true, but, on the contrary, any value of $x$ which satisfies one of the quadratics will render the other $=A$, some numerical quantity differing from 0 , we in fact collect the product of

$$
\begin{aligned}
& x^{2}-3 x+2=0, \text { or } A, \\
& \text { by } \quad x^{2}+7 x+12=\mathrm{A} \text {, or } 0,
\end{aligned}
$$

in finding $\left(x^{2}-3 x+2\right)\left(x^{2}+7 x+12\right)=0$; where the premises being strictly correct, the result is unexceptionable. And the same result arises, although not with equally clear evidence of its truth, when $A$ is superseded by zero.

The same test, of a bypothetical adjustment of one of the two proposed equations, would at once expose the fallacy of each of the conclusions attained in our imaginary experiment.

The general propriety of keeping the zero-side of each equation in a chain of argument clear from any transposed terms, is proved therefore by the liberty which it allows to the mind, of conceiving any zero, which happens to be pro tempore incorrect, to be superseded by the correct valne, and of perceiving without any embarrassment or additional labour the exact conditions of the final result. But the especial propriety of adhering to this expedient, when surds are to be extricated, appears in the necessity which it imposes of attending to the copula of the argument, the suppression of which in the vulgar process occasions all the obscurity that is complained of. 'Thus, between the statements

$$
\begin{aligned}
& a=\sqrt{ } x \text { or } a-\sqrt{ } x=0 \\
& a^{2}=x \text { or } a^{2}-x=0
\end{aligned}
$$


the copula $a+\sqrt{ } x=\mathrm{A}$ has been lost sight of. The complete chain is

$$
\begin{aligned}
a-\sqrt{ } x & =0 \text { or } \mathrm{A} \\
a+\sqrt{ } x & =A_{1} \text { or } 0 \\
\therefore \quad a^{2}-x & =0 .
\end{aligned}
$$

You are well aware that this copula will, in all cases of surd equations, consist of all the variations that can be made of the given formula by varying the affection of each radical it contains in all possible ways. You also can refer, more readily than myself, to various authors in whose works the method of forming the continued product of a formula and all such variations of it (for the sake of a convenient term I would venture to say, its congeners) has been simplified. You see, that by retaining the entire set of congeneric equations, all doubt. respecting the constancy of every symbol employed, whether letter or radical sign, is entirely cleared away. Uncertainty, indeed, still remains attached to the results of the solution of the final equation; namely, uncertainty as to which of the congeneric formulæ will be reduced to zero, by the resulting values of $x$; but this doubt is unconnected with any perplexity respecting the general theory.

A very unnecessary ambiguity is admitted in the current acceptation of the word root; and great advantage would accrue from restricting it to its only legitimate signification of " such a value of the unknown in any linear divisor of the equation, as will cause that divisor to vanish."

The sum of the whole matter, respecting surd equations, I conceive to be this. We know that the continued product of a surd formula and all its congeners will produce a rational formula; and that such rational formula, being equated to zero, may be solved by as many roots as it has dimensions. We are also certain thai each of these roots will cause one of the congeneric surd formulæ to vanish; otherwise the product of all would not be $=0$ as assumed. But, is the value of $x$ which effects this, to be called a root of the surd formula? No, it is a root of the rational combination only. - Have irrational equations, then, no roots? None at all.-What have they, then, in the place of roots? An equitable chance, in common with each formula in the congeneric society, of solution by means of the solution of the stock-equation.-But if an equation has no root, nor even a certainty of solution, in what form can it be intelligibly proposed? A note of interrogation subjoined might serve to intimate that the equation is proposed either for solution or correction.-To what order can surd equations be assigned? 'To the fractional order $\frac{n}{n}$, when

Third Series. Vol. 8. No.43. Jan. 1836. 
50 Letter from the Rev. Dr. Lardner to Peter Barlow, Esq.

$n$ congeneric formulæ produce a rational equation of the $m$ th order; thus,

$$
\begin{aligned}
& 2 x+\sqrt{x^{2}-a}=b ? \text { is of the } \frac{2}{2} \text { order. } \\
& a+\sqrt{x+\sqrt{x-b} ?=0}=0 \text { ? } \\
& a \sqrt[3]{4} \ldots \ldots \\
& a+x-\sqrt[3]{a-x}=0 ? \quad \frac{6}{9} \ldots \ldots
\end{aligned}
$$

Are the chances of solution equal for each individual congener? I leave that question in good hands, and remain,

Yours, very affectionately,

Bath, Nov. 12, 1835.

W. G. Honner.

XI. Letter to Peter Barlow, Esq., F.R.S., \&c., \&c., respecting some parts of his Reports addressed to the Directors and Proprietors of the London and Birmingham Railway Company. By the Rev. Dionysius Lardner, LL.D., F.R.S., \&c.

Dear Sir,

I $T$ was not until my return to London within the last few days that $I$ had the pleasure of receiving a copy of your Second Report addressed to the Directors of the London and Birmingham Railway Company. The previous communications which had passed led me to anticipate some collision of opinion between us, but I confess I did not expect that any difference should exist on a question of a nature so elementary as that which you have noticed in your Report. In page 87 you say:

"If (as was assumed in the Parliamentary Committee on the question of the Great Western Railway) as much power was gained in the descent as was lost in the ascent, the odds would be made all even. But that assumption is altogether erroneous both in theory and practice."

And again, in page 91, you say, referring to your theory of the deflexion of bars:

"The only doubt, therefore, which can remain is, how far I ought to reject as inconsiderable any increase of power on the descending side. This point cannot be met experimentally, and I am therefore obliged to depend here only on demonstration. The case certainly involves no difficulty of conception to those acquainted with theoretical mechanics; but the question having been seen in a different light by a gentleman of considerable scientific eminence, I should have been glad to have exhibited the effect experimentally; but as the whole turns upon velocity, this is of course impossible."

To those who have taken an interest in the question respect- 